\title{
YY Canis Minoris: Contact or near contact system? $?^{\star, \star \star}$
}

\author{
P.G. Niarchos ${ }^{1}$, L. Mantegazza $^{2}$, E. Poretti ${ }^{2}$, and V. Manimanis ${ }^{1}$ \\ 1 Section of Astrophysics, Department of Physics, University of Athens, Panepistimiopolis, GR-15784 Zografos, Athens, Greece \\ 2 Osservatorio Astronomico di Brera, Via Bianchi 46, 23807 Merate (LC), Italy
}

Received February 12; accepted May 14, 1998

\begin{abstract}
New $V$ photoelectric observations of the eclipsing system YY CMi, obtained at La Silla, Chile, and Merate Observatory, Italy, are presented. New times of minima and ephemeris based on our observations are also given. The $V$ light curve was analysed by using the WD code to derive the geometrical and physical parameters of the system. Since no spectroscopic mass ratio is available, the $q$-search method was applied to yield the preliminary range of the mass ratio in order to search for the final solution. First the unspotted solution was carried out by using the unperturbed parts of the light curve and applying the DC program of the WD code. The solution was performed by assuming contact (mode 3) and semi-detached (mode 4) configuration, since no classification of the system is possible from the shape of the light curve. The solution in mode 4 does not lead to an acceptable model, since the secondary was found to be slightly overcontact. Therefore the contact solution was finally adopted. Moreover the light curve peculiarities (Max II fainter than Max I and excess of light around the phase 0.32 ) were explained by assuming a cool and a hot spot on the surface of the secondary (cooler) component. The degree of contact is very small $(f \approx 3 \%)$ and the thermal contact is poor $\left(T_{1}-T_{2}\right) \approx 650 \mathrm{~K}$. These results together with the high photometric mass ratio $q \approx 0.89$ indicate that YY CMi is very probably a system at the beginning or the end of the contact phase.
\end{abstract}

Key words: stars: YY CMi — binaries: eclipsing starspots

Send offprint requests to: P.G. Niarchos

* Based on observations partly made at the European Southern Observatory (ESO).

** Table 1 is only available in electronic form at the CDS via anonymous ftp to cdsarc.u-strasbg.fr (130.79.128.5) or via http://cdsweb.u-strasbg.fr/Abstract.html

\section{Introduction}

The light variability of YY CMi ( $\equiv$ HD67100) was discovered by Morgenroth (1934). Later photometric observations were due to Lause (1938), Soloviev (1940), Kaho (1950), and Kordylewsky \& Szafraniec (1957). According to the General Catalogue of Variable Stars (GCVS, III ed., Kukarkin et al. 1969), the system is classified as a $\beta$ Lyrae type eclipsing binary with a period of $1.0940253 \mathrm{~d}$ and a spectral type F5. Later, the spectrum was classed by Hill et al. (1975) as F6V at phase 0.31 and F7V at phase 0.71 . The first complete light curve in three colours $(u, b, y)$ was obtained by Abhyankar (1962), who also presented a solution based on Russell and Merrill method. From a questionable treatment of the colour indices, Abhyankar (1962) concluded that the system is composed of an F6III primary and an A5V secondary. Koch et al. (1970) noticed that YY CMi was probably a system of two (F5 + F8) main sequence stars.

Giuricin \& Mardirossian (1981) reanalyzed Abhyankar's (1962) three-colour photoelectric observations by using Wood's (1972) model and found a solution appreciably different from the previous ones. The elements they derived lead to an evolved contact system consisting of a primary (roughly an F6 star) and a secondary (early G5) of practically equal sizes. This picture of the system is only an approximation of the real one, since Wood's model treats the stars as triaxial ellipsoids and does not handle contact systems very well.

\section{Observations}

YY CMi was observed in the framework of a two-site campaign (European Southern Observatory, La Silla, Chile and Merate Observatory, Italy) devoted to the $\delta$ Scuti star BI CMi (Mantegazza \& Poretti 1994). The 277 ESO observations cover 14 consecutive nights (from JD 2448280 to JD 2448293), while the Merate ones are distributed over 8 nights (from JD 2448273 to JD 2448291). We have observations from the two sites in the same night for 5 cases; 
Table 1. Individual $V$ observations of YY CMi

\begin{tabular}{|c|c|c|c|c|c|c|c|c|c|c|c|}
\hline $\begin{array}{c}\text { HJD } \\
2448200+\end{array}$ & $\Delta V$ & $\begin{array}{c}\text { HJD } \\
2448200+\end{array}$ & $\Delta V$ & $\begin{array}{c}\text { HJD } \\
2448200+\end{array}$ & $\Delta V$ & $\begin{array}{c}\text { HJD } \\
2448200+\end{array}$ & $\Delta V$ & $\begin{array}{c}\text { HJD } \\
2448200+\end{array}$ & $\Delta V$ & $\begin{array}{c}\text { HJD } \\
2448200+\end{array}$ & $\Delta V$ \\
\hline 73.4463 & 2.363 & 80.4971 & 1.886 & 82.8047 & 2.064 & 85.6123 & 1.835 & 88.7217 & 2.093 & 90.5918 & 1.738 \\
\hline 73.4629 & 2.424 & 80.5039 & 1.907 & 82.8145 & 2.024 & 85.6230 & 1.804 & 88.7305 & 2.156 & 90.6143 & 1.716 \\
\hline 73.4688 & 2.430 & 80.5078 & 1.939 & 82.8252 & 1.984 & 85.6787 & 1.702 & 88.7412 & 2.227 & 90.6230 & 1.710 \\
\hline 73.4736 & 2.423 & 80.5146 & 1.949 & 82.8359 & 1.939 & 85.6875 & 1.696 & 88.7803 & 2.429 & 90.6318 & 1.704 \\
\hline 73.4854 & 2.367 & 80.5215 & 2.001 & 82.8486 & 1.907 & 85.6963 & 1.690 & 88.7891 & 2.416 & 90.6396 & 1.698 \\
\hline 73.4941 & 2.308 & 80.5283 & 2.021 & 83.5898 & 1.629 & 85.7070 & 1.674 & 88.7988 & 2.375 & 90.6699 & 1.679 \\
\hline 73.5049 & 2.228 & 80.5391 & 2.081 & 83.5986 & 1.641 & 85.7275 & 1.655 & 88.8105 & 2.293 & 90.6787 & 1.681 \\
\hline 73.5117 & 2.192 & 80.5439 & 2.101 & 83.6074 & 1.638 & 85.7383 & 1.644 & 88.8213 & 2.224 & 90.6885 & 1.680 \\
\hline 73.5186 & 2.148 & 80.5508 & 2.121 & 83.6152 & 1.646 & 85.7471 & 1.646 & 88.8320 & 2.142 & 90.7031 & 1.679 \\
\hline 73.5293 & 2.058 & 80.5557 & 2.139 & 83.6240 & 1.642 & 85.7754 & 1.644 & 88.8447 & 2.061 & 90.7109 & 1.680 \\
\hline 73.5352 & 2.025 & 80.5654 & 2.163 & 83.6328 & 1.651 & 85.7861 & 1.636 & 89.5400 & 1.697 & 90.7188 & 1.683 \\
\hline 73.5400 & 1.993 & 80.5723 & 2.160 & 83.6572 & 1.669 & 85.7949 & 1.636 & 89.5488 & 1.693 & 90.7363 & 1.687 \\
\hline 73.5479 & 1.960 & 80.5791 & 2.173 & 83.6807 & 1.678 & 85.8066 & 1.642 & 89.5693 & 1.688 & 90.7432 & 1.692 \\
\hline 73.5547 & 1.922 & 80.5889 & 2.157 & 83.6895 & 1.685 & 85.8164 & 1.650 & 89.5781 & 1.682 & 90.7520 & 1.698 \\
\hline 73.5645 & 1.872 & 80.5938 & 2.155 & 83.6992 & 1.706 & 85.8291 & 1.652 & 89.5869 & 1.676 & 90.7715 & 1.710 \\
\hline 73.5742 & 1.850 & 80.6084 & 2.091 & 83.7080 & 1.714 & 85.8408 & 1.662 & 89.5967 & 1.680 & 90.7793 & 1.716 \\
\hline 73.5898 & 1.796 & 80.6172 & 2.071 & 83.7305 & 1.741 & 86.5840 & 2.415 & 89.6172 & 1.677 & 90.7891 & 1.740 \\
\hline 73.6064 & 1.780 & 80.6250 & 2.034 & 83.7383 & 1.758 & 86.5947 & 2.438 & 89.6348 & 1.682 & 90.7988 & 1.740 \\
\hline 74.3906 & 1.729 & 80.6523 & 1.935 & 83.7471 & 1.784 & 86.6035 & 2.413 & 89.6465 & 1.690 & 90.8086 & 1.749 \\
\hline 74.4023 & 1.747 & 80.6592 & 1.914 & 83.7559 & 1.802 & 86.6143 & 2.360 & 89.6563 & 1.696 & 90.8193 & 1.763 \\
\hline 74.4160 & 1.776 & 80.6660 & 1.886 & 83.7646 & 1.827 & 86.6221 & 2.311 & 89.6719 & 1.711 & 90.8311 & 1.793 \\
\hline 74.4258 & 1.790 & 80.6953 & 1.808 & 83.7900 & 1.929 & 86.6309 & 2.254 & 89.6797 & 1.717 & 90.8447 & 1.824 \\
\hline 74.4326 & 1.819 & 80.7051 & 1.789 & 83.7998 & 1.969 & 86.6465 & 2.134 & 89.6885 & 1.725 & 90.8584 & 1.873 \\
\hline 74.4473 & 1.849 & 80.7129 & 1.773 & 83.8105 & 2.019 & 86.6592 & 2.040 & 89.6963 & 1.736 & 91.3711 & 1.692 \\
\hline 74.4561 & 1.885 & 80.7363 & 1.749 & 83.8213 & 2.072 & 86.6826 & 1.923 & 89.7178 & 1.762 & 91.3838 & 1.728 \\
\hline 74.4658 & 1.928 & 80.7441 & 1.735 & 83.8330 & 2.104 & 86.6934 & 1.879 & 89.7256 & 1.772 & 91.3916 & 1.736 \\
\hline 74.4727 & 1.950 & 80.7549 & 1.730 & 83.8447 & 2.157 & 86.7041 & 1.841 & 89.7432 & 1.798 & 91.4258 & 1.829 \\
\hline 74.4805 & 2.001 & 80.7656 & 1.719 & 84.3760 & 2.293 & 86.7139 & 1.813 & 89.7656 & 1.867 & 91.4287 & 1.848 \\
\hline 74.4941 & 2.072 & 80.7793 & 1.713 & 84.3838 & 2.385 & 86.7344 & 1.760 & 89.7744 & 1.899 & 91.5371 & 2.139 \\
\hline 74.5059 & 2.140 & 80.7891 & 1.690 & 84.4023 & 2.425 & 86.7490 & 1.740 & 89.7822 & 1.918 & 91.5469 & 2.111 \\
\hline 74.5107 & 2.167 & 80.8135 & 1.686 & 84.4150 & 2.420 & 86.7822 & 1.699 & 89.7910 & 1.944 & 91.5576 & 2.067 \\
\hline 74.5215 & 2.258 & 80.8271 & 1.681 & 84.4199 & 2.390 & 86.7969 & 1.675 & 89.8008 & 2.006 & 91.5664 & 2.032 \\
\hline 74.5273 & 2.311 & 80.8408 & 1.675 & 84.4287 & 2.349 & 86.8066 & 1.667 & 89.8105 & 2.062 & 91.5762 & 1.988 \\
\hline 74.5371 & 2.369 & 81.5938 & 1.890 & 84.4375 & 2.293 & 86.8184 & 1.658 & 89.8223 & 2.136 & 91.5947 & 1.916 \\
\hline 74.5488 & 2.411 & 81.6025 & 1.928 & 84.4473 & 2.217 & 86.8301 & 1.654 & 89.8369 & 2.237 & 91.6094 & 1.876 \\
\hline 74.5547 & 2.440 & 81.6123 & 1.974 & 84.4541 & 2.157 & 86.8438 & 1.649 & 89.8496 & 2.329 & 91.7744 & 1.677 \\
\hline 74.5625 & 2.465 & 81.6211 & 2.012 & 84.4629 & 2.108 & 87.5811 & 1.868 & 89.8604 & 2.369 & 91.7842 & 1.681 \\
\hline 74.5674 & 2.438 & 81.6289 & 2.050 & 84.4736 & 2.041 & 87.5908 & 1.912 & 90.3613 & 1.940 & 91.7939 & 1.677 \\
\hline 74.5742 & 2.426 & 81.6377 & 2.085 & 84.4814 & 1.985 & 87.6006 & 1.951 & 90.3691 & 1.968 & 91.8047 & 1.678 \\
\hline 74.5879 & 2.329 & 81.6475 & 2.116 & 84.4883 & 1.949 & 87.6104 & 1.987 & 90.3740 & 2.000 & 91.8174 & 1.684 \\
\hline 74.5938 & 2.297 & 81.6563 & 2.148 & 84.4932 & 1.934 & 87.6182 & 2.035 & 90.3848 & 2.036 & 91.8301 & 1.688 \\
\hline 74.5977 & 2.254 & 81.7021 & 2.098 & 84.5010 & 1.896 & 87.6289 & 2.096 & 90.3926 & 2.075 & 91.8438 & 1.691 \\
\hline 74.6035 & 2.214 & 81.7148 & 2.046 & 84.5166 & 1.842 & 87.6445 & 2.216 & 90.4004 & 2.102 & 91.8574 & 1.700 \\
\hline 77.3975 & 1.846 & 81.7344 & 1.973 & 84.5186 & 1.839 & 87.6533 & 2.266 & 90.4053 & 2.112 & 92.5342 & 1.886 \\
\hline 77.4063 & 1.823 & 81.7422 & 1.944 & 84.5264 & 1.806 & 87.6621 & 2.329 & 90.4121 & 2.144 & 92.5625 & 2.018 \\
\hline 77.4121 & 1.809 & 81.7510 & 1.913 & 84.5371 & 1.807 & 87.6826 & 2.421 & 90.4141 & 2.151 & 92.5723 & 2.061 \\
\hline 77.4297 & 1.767 & 81.7607 & 1.878 & 84.5459 & 1.758 & 87.6934 & 2.424 & 90.4209 & 2.150 & 92.5811 & 2.089 \\
\hline 77.4414 & 1.756 & 81.7695 & 1.852 & 84.5850 & 1.706 & 87.7041 & 2.378 & 90.4277 & 2.149 & 92.6055 & 2.167 \\
\hline 77.4492 & 1.743 & 81.7988 & 1.791 & 84.5938 & 1.697 & 87.7275 & 2.222 & 90.4336 & 2.145 & 92.6133 & 2.172 \\
\hline 77.4590 & 1.730 & 82.3770 & 1.717 & 84.6035 & 1.684 & 87.7383 & 2.158 & 90.4414 & 2.127 & 92.6201 & 2.165 \\
\hline 77.4736 & 1.719 & 82.3867 & 1.706 & 84.6133 & 1.672 & 87.7490 & 2.077 & 90.4453 & 2.120 & 92.6328 & 2.139 \\
\hline 77.4893 & 1.704 & 82.3877 & 1.710 & 84.6211 & 1.664 & 87.7744 & 1.932 & 90.4512 & 2.115 & 92.6436 & 2.097 \\
\hline 77.4971 & 1.709 & 82.4736 & 1.638 & 84.6309 & 1.657 & 87.7852 & 1.889 & 90.4580 & 2.080 & 92.6631 & 2.024 \\
\hline 77.5039 & 1.702 & 82.4873 & 1.629 & 84.6396 & 1.653 & 87.7979 & 1.845 & 90.4639 & 2.047 & 92.6729 & 1.987 \\
\hline 77.5156 & 1.689 & 82.5000 & 1.628 & 84.6611 & 1.642 & 87.8105 & 1.802 & 90.4707 & 2.024 & 92.6924 & 1.906 \\
\hline 77.5273 & 1.691 & 82.5244 & 1.642 & 84.6826 & 1.640 & 87.8232 & 1.774 & 90.4746 & 2.009 & 92.7002 & 1.887 \\
\hline 77.5332 & 1.690 & 82.5381 & 1.644 & 84.6904 & 1.633 & 87.8369 & 1.761 & 90.4795 & 1.998 & 92.7100 & 1.858 \\
\hline 77.5371 & 1.684 & 82.5527 & 1.667 & 84.6992 & 1.638 & 87.8516 & 1.727 & 90.4834 & 1.970 & 92.7314 & 1.800 \\
\hline
\end{tabular}


Table 1. continued

\begin{tabular}{|c|c|c|c|c|c|c|c|c|c|c|c|}
\hline HJD & $\Delta V$ & HJD & $\Delta V$ & HJD & $\Delta V$ & HJD & $\Delta V$ & HJD & $\Delta V$ & HJD & $\Delta V$ \\
\hline $2448200+$ & & $2448200+$ & & $2448200+$ & & $2448200+$ & & $2448200+$ & & $2448200+$ & \\
\hline 77.5498 & 1.679 & 82.6299 & 1.733 & 84.7080 & 1.641 & 88.5439 & 1.690 & 90.4902 & 1.949 & 92.7803 & 1.730 \\
\hline 77.5605 & 1.681 & 82.6396 & 1.751 & 84.7334 & 1.656 & 88.5547 & 1.688 & 90.4961 & 1.934 & 92.7900 & 1.732 \\
\hline 77.5693 & 1.665 & 82.6484 & 1.768 & 84.7422 & 1.657 & 88.5635 & 1.699 & 90.5049 & 1.905 & 92.8008 & 1.721 \\
\hline 77.5859 & 1.690 & 82.6650 & 1.818 & 84.7520 & 1.663 & 88.5859 & 1.715 & 90.5088 & 1.883 & 92.8154 & 1.708 \\
\hline 77.5967 & 1.686 & 82.6738 & 1.838 & 84.7646 & 1.679 & 88.5967 & 1.733 & 90.5166 & 1.855 & 92.8291 & 1.695 \\
\hline 80.3955 & 1.669 & 82.6934 & 1.917 & 84.7891 & 1.696 & 88.6074 & 1.739 & 90.5205 & 1.852 & 92.8438 & 1.685 \\
\hline 80.4131 & 1.705 & 82.7021 & 1.955 & 84.7979 & 1.706 & 88.6162 & 1.755 & 90.5264 & 1.860 & 92.8574 & 1.680 \\
\hline 80.4268 & 1.706 & 82.7109 & 1.996 & 84.8076 & 1.718 & 88.6367 & 1.778 & 90.5303 & 1.851 & 93.5361 & 1.691 \\
\hline 80.4453 & 1.730 & 82.7207 & 2.031 & 84.8174 & 1.733 & 88.6572 & 1.826 & 90.5352 & 1.828 & 93.5488 & 1.706 \\
\hline 80.4502 & 1.746 & 82.7412 & 2.120 & 84.8262 & 1.747 & 88.6660 & 1.845 & 90.5420 & 1.811 & 93.5576 & 1.713 \\
\hline 80.4600 & 1.748 & 82.7500 & 2.154 & 84.8389 & 1.771 & 88.6846 & 1.906 & 90.5479 & 1.796 & 93.5664 & 1.727 \\
\hline 80.4688 & 1.790 & 82.7588 & 2.169 & 85.5850 & 1.937 & 88.6934 & 1.944 & 90.5508 & 1.792 & & \\
\hline 80.4824 & 1.833 & 82.7676 & 2.168 & 85.5938 & 1.898 & 88.7031 & 1.984 & 90.5586 & 1.784 & & \\
\hline 80.4902 & 1.857 & 82.7949 & 2.103 & 85.6035 & 1.870 & 88.7129 & 2.041 & 90.5840 & 1.742 & & \\
\hline
\end{tabular}

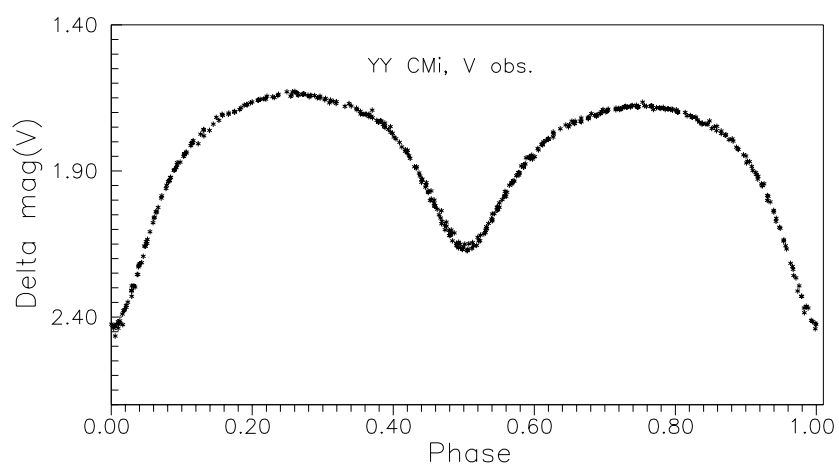

Fig. 1. The individual $V$ observations of YY CMi

since on these nights there is a partial superimposition of the observations for almost two hours, it was possible to get an excellent alignment between the two datasets. The individual $V$ observations are given in Table 1 and the respective light curve is shown in Fig. 1.

We have performed differential photometry in the $V$ band with respect to the two comparison stars HD 66925 and HD 67028; since the light variability of the $\delta$ Sct star $\mathrm{BI} \mathrm{CMi}$ was faster than that of YY CMi, the latter was measured once every five cycles. The comparison of the differential magnitudes between the two comparison stars has shown, as expected, a different accuracy between the data gathered at La Silla and at Merate: the former have a mean standard deviation of $4.4 \mathrm{mmag}$ for each measurement, against the $8.6 \mathrm{mmag}$ for the latter (a value quite high due to unfavourable declination of the field with respect to the latitude of Merate Observatory). Moreover, Mantegazza \& Poretti (1994) discussed the possible microvariability of HD 67028.

\section{The period of the system from the times of minima}

The present observations, obtained at Merate Observatory and ESO, were used to calculate new times of minima by
Table 2. New times of minima of YY CMi

\begin{tabular}{lccrc}
\hline $\begin{array}{l}\text { JD Hel. } \\
2440000\end{array}$ & Error & $E$ & O - C & $\begin{array}{c}\text { Type of } \\
\text { minimum }\end{array}$ \\
\hline 8273.4650 & 0.0050 & -13.0 & 0.0007 & I \\
8274.5561 & 0.0006 & -12.0 & -0.0020 & I \\
8280.5688 & 0.0082 & -6.5 & -0.0052 & II \\
8281.6774 & 0.0044 & -5.5 & 0.0097 & II \\
8282.7544 & 0.0041 & -4.5 & -0.0071 & II \\
8284.4080 & 0.0050 & -3.0 & 0.0058 & I \\
8287.6896 & 0.0030 & 0.0 & 0.0060 & I \\
8288.7719 & 0.0023 & 1.0 & -0.0055 & I \\
8290.4151 & 0.0018 & 2.5 & -0.0029 & II \\
8292.6061 & 0.0007 & 4.5 & 0.0005 & II \\
\hline
\end{tabular}

using Kwee \& Van Woerden (KW) method. The new minima times are given in Table 2. The successive columns give the HJD of minimum, the error, the number of cycles $E$, the $(\mathrm{O}-\mathrm{C})$ values and the type of minimum (I: primary, II: secondary). A least-squares solution, applied to all minima listed in Table 2, yields the following ephemeris

$$
\begin{gathered}
\text { J.D.Hel. }(\text { Min I })=2448287.6836+1.0937869 \cdot E \\
\pm 0.0023 \pm 0.0003484
\end{gathered}
$$

which has been used for the calculation of $\mathrm{O}-\mathrm{C}$ values.

The $\mathrm{O}-\mathrm{C}$ behaviour for all the existing minima times, computed by using the above ephemeris, is shown in Fig. 2. The existing data are not enough to draw definite conclusions about the variation of the period. The GCSV, IV ed., (Kholopov et al. 1985) gives a period $1.0940197 \mathrm{~d}$. This period was calculated by Abhyankar (1962) using well determined minima times over a period of 24 years. Our new ephemeris, based on the present observations, suggests a shorter period. 


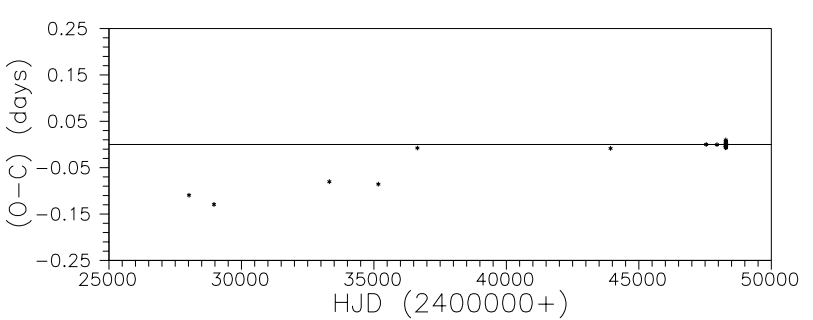

Fig. 2. The $\mathrm{O}-\mathrm{C}$ diagram of $\mathrm{YY} \mathrm{CMi}$

\section{Light curve analysis}

The light curve analysis is quite difficult for the following reasons: (a) no spectroscopic mass-ratio is known; (b) the maxima of the light curve are unequal in brigthness (Max I brighter than Max II); (c) the system undergoes only partial eclipses. An inspection of the light curve reveals that brightness variations occur not only around the maxima, but also at other phases. More specifically, a decrease in brightness is present in the phase interval $0.59-0.87$ and a small excess of light is seen around phase 0.32 . Other minor light variations can be seen in other phase regions. The magnitude difference between the two maxima is about Max II - Max I = 0.03 mag. In modelling light curves of systems exhibiting light curve anomalies, the need to place hot and/or cool spots of solar type has been suggested by several investigators (e.g. Binnendijk 1960; Hilditch 1981; Linnell 1982; Van Hamme \& Wilson 1985; Milone et al. 1987; van't Veer \& Maceroni 1988, 1989; Maceroni et al. 1990).

\subsection{Unspotted solution}

The most recent (1996) version of the Wilson-Devinney (Wilson 1990) synthetic light curve code was used for the light curve solution. 66 normal points, listed in Table 3, were used and weights equal to the number of observations per normal were assigned. Both unspotted and spotted solutions were performed; for the latter, we assumed the presence of cool and hot spots to explain the difference in brightness between the two maxima and the excess of light, respectively. Under these assumptions we excluded the observations in the phase interval $0.59-0.87$ from the unspotted solution, since a significant decrease of brightness occurs.

The subscripts 1 and 2 refer to the component eclipsed at primary and secondary minimum, respectively. A preliminary set of input parameters for the DC program was obtained by the Binary Maker 2.0 program (Bradstreet 1993). The DC program was used in the contact mode 3 and in the semidetached mode 4 . In the subsequent analysis the following assumptions were made: a mean surface temperature $T_{1}=6360 \mathrm{~K}$ according to the spectral type $\mathrm{F} 6 \mathrm{~V}$; we assigned typical values for stars with convective envelopes to bolometric albedos and gravity darkening
Table 3. Normal points for YY CMi in light units

\begin{tabular}{cccccc}
\hline phase & $l_{\mathrm{V}}$ & $n$ & phase & $l_{\mathrm{V}}$ & $n$ \\
\hline 0.00783 & 0.48137 & 11.0 & 0.50773 & 0.61695 & 8.0 \\
0.02287 & 0.51976 & 10.0 & 0.52366 & 0.63518 & 7.0 \\
0.03789 & 0.57334 & 10.0 & 0.53646 & 0.66575 & 10.0 \\
0.05239 & 0.63842 & 8.0 & 0.55349 & 0.71225 & 12.0 \\
0.06562 & 0.69908 & 6.0 & 0.56933 & 0.75813 & 6.0 \\
0.08267 & 0.76389 & 9.0 & 0.58190 & 0.78970 & 11.0 \\
0.09990 & 0.81603 & 10.0 & 0.59689 & 0.82158 & 7.0 \\
0.11422 & 0.85608 & 5.0 & 0.61420 & 0.85730 & 9.0 \\
0.12828 & 0.87877 & 5.0 & 0.62881 & 0.88346 & 4.0 \\
0.14416 & 0.90881 & 3.0 & 0.64715 & 0.90434 & 3.0 \\
0.15985 & 0.93352 & 5.0 & 0.65910 & 0.91634 & 6.0 \\
0.17454 & 0.94462 & 3.0 & 0.67556 & 0.92649 & 5.0 \\
0.18769 & 0.96072 & 5.0 & 0.69124 & 0.93876 & 8.0 \\
0.20482 & 0.97750 & 5.0 & 0.70327 & 0.94778 & 4.0 \\
0.21864 & 0.98643 & 3.0 & 0.71938 & 0.95337 & 6.0 \\
0.23310 & 0.99242 & 4.0 & 0.73380 & 0.96041 & 8.0 \\
0.25381 & 1.00000 & 4.0 & 0.74856 & 0.96248 & 7.0 \\
0.26586 & 0.99982 & 6.0 & 0.76533 & 0.95914 & 5.0 \\
0.27942 & 0.99460 & 5.0 & 0.77979 & 0.95545 & 5.0 \\
0.29477 & 0.98801 & 5.0 & 0.79565 & 0.95080 & 7.0 \\
0.30943 & 0.97813 & 4.0 & 0.80974 & 0.94060 & 4.0 \\
0.32286 & 0.96843 & 3.0 & 0.82615 & 0.92802 & 5.0 \\
0.34133 & 0.96221 & 3.0 & 0.84137 & 0.91143 & 6.0 \\
0.35564 & 0.94087 & 6.0 & 0.85597 & 0.89722 & 4.0 \\
0.37072 & 0.92964 & 7.0 & 0.86969 & 0.87827 & 5.0 \\
0.38562 & 0.90732 & 9.0 & 0.88500 & 0.85045 & 5.0 \\
0.40026 & 0.87768 & 6.0 & 0.90195 & 0.80985 & 6.0 \\
0.41852 & 0.83159 & 7.0 & 0.91745 & 0.76525 & 8.0 \\
0.43452 & 0.78076 & 6.0 & 0.93045 & 0.72516 & 5.0 \\
0.44748 & 0.74103 & 10.0 & 0.94591 & 0.66138 & 8.0 \\
0.46273 & 0.69124 & 11.0 & 0.96274 & 0.58903 & 6.0 \\
0.47808 & 0.64879 & 10.0 & 0.97808 & 0.52715 & 8.0 \\
0.49287 & 0.62114 & 10.0 & 0.99542 & 0.48774 & 7.0 \\
\hline & & & & & \\
& & & & \\
0.04 &
\end{tabular}

coefficients; limb darkening coefficients were taken from Al-Naimiy's (1978) tables and bolometric linear limb darkening coefficients from Van Hamme (1993). Third light was assumed to be $\ell_{3}=0$. The adjustable parameters were: the phase of conjunction $\phi_{0}$, the inclination $i$, the temperature $T_{2}$, the nondimensional potential $\Omega_{1}$ in mode 3 and $\Omega_{2}$ in mode 4 , the monochromatic luminosity $L_{1}$ and the mass-ratio $q=m_{2} / m_{1}$.

Since no spectroscopic mass-ratio of the system is known, a search for the solution was made for a massratio $q$ ranging from 0.2 to 4 . The lowest values of the sum $\Sigma(\text { res })^{2}$ of the weighted squared residuals occured around $q=1.0$ in mode 3 and $q=0.8$ in mode 4 . Figure 3 shows the fit parameters $\Sigma(\text { res })^{2}$ as a function of the mass-ratio $q$ in modes 3 and 4 . In order to find the final unspotted solution we continued the analysis by applying the DC program for both cases. The two solutions converged to $q=0.8921$ in mode 3 and $q=0.8295$ in mode 4 . The corresponding values of $\Sigma$ (res) ${ }^{2}$ were found to be 0.0785 and 0.0835 , respectively. Of these two solutions, we finally adopted the solution in mode 3 (with $q=0.8921$ ) by 


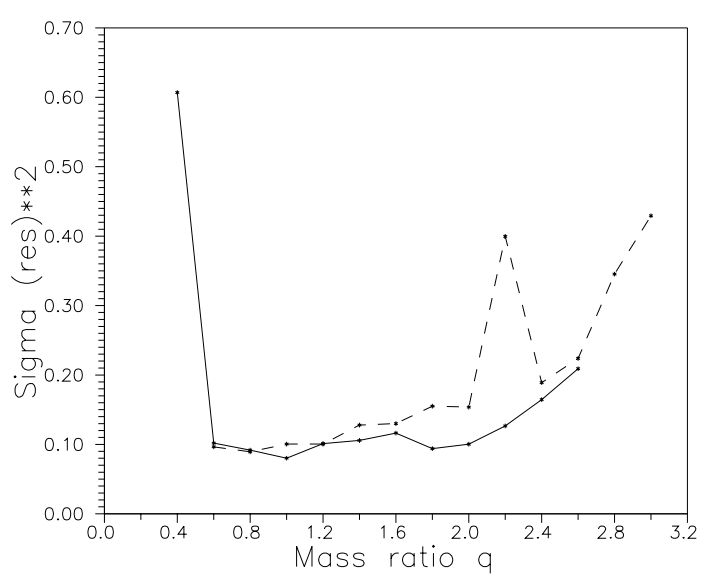

Fig. 3. The fit parameter $\Sigma(\text { res })^{2}$ as a function of the mass-ratio $q$. Solid lines: mode 3 ; dashed lines: mode 4

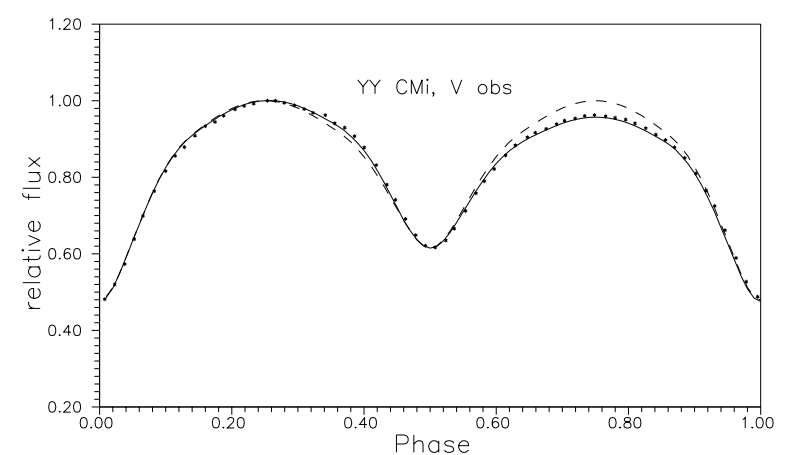

Fig. 4. Normal points and theoretical $V$ light curves of YY CMi. Dashed lines: unspotted solution; solid lines: spotted solution

taking into account the better fit of the solution in mode 3 and the fact that the secondary exceeds the Roche lobe $\left(\Omega_{2}<\Omega_{\text {in }}\right)$ in mode 4 . The results of the unspotted solution are given in Table 4 and the corresponding theoretical light curves are shown as dashed lines in Fig. 4.

\subsection{Spotted solution}

The spotted solution was carried out by adopting the simplest spot model with a physical meaning. We started by assuming that the system had a cool spot on the secondary (cooler) component of the same nature as solar magnetic spots (Mullan 1975). Such a spot could explain the decrease of brightness in the phase interval 0.59-0.87. Another hot spot was assumed on the secondary component near the neck region in order to match the light excess around phase 0.32 . Such a bright region can be explained as a result of energy transfer from the primary to the secondary component (Van Hamme \& Wilson 1985).

The Binary Maker 2.0 program was used to obtain the best fit by adjusting the spot parameters: the latitude $b$, the longitude $l$, the angular radius $R$ and the temperature factor T.F. Once the best fit was obtained, the DC program was used to derive the final solution. The program
Table 4. Light curve solutions of YY CMi

\begin{tabular}{lcc}
\hline Parameter & $\begin{array}{c}\text { unspotted } \\
\text { solution }\end{array}$ & $\begin{array}{c}\text { spotted } \\
\text { solution }\end{array}$ \\
\hline$\phi_{0}$ & $0.0026 \pm 0.0003$ & $0.0026 \pm 0.0003$ \\
$i$ (degrees) & $79.50 \pm 0.16$ & $79.47 \pm 0.07$ \\
$g_{1}\left(=g_{2}\right)$ & $0.32^{*}$ & $0.32^{*}$ \\
$T_{1}(\mathrm{~K})$ & $6360^{*}$ & $6360^{*}$ \\
$T_{2}(\mathrm{~K})$ & $5707 \pm 11$ & $5710 \pm 4$ \\
$A_{1}\left(=A_{2}\right)$ & $0.5^{*}$ & $0.5^{*}$ \\
$\Omega_{1}\left(=\Omega_{2}\right)$ & $3.558 \pm 0.013$ & $3.560 \pm 0.006$ \\
$q=m_{2} / m_{1}$ & $0.892 \pm 0.011$ & $0.885 \pm 0.004$ \\
$L_{1} /\left(L_{1}+L_{2}\right)(V)$ & $0.641 \pm 0.003$ & $0.642 \pm 0.002$ \\
$x_{1}\left(=x_{2}\right)(V)$ & $0.60^{*}$ & $0.60^{*}$ \\
$x_{1}\left(=x_{2}\right)($ bolo $)$ & $0.50^{*}$ & $0.50^{*}$ \\
$\%$ overcontact & $3 \%$ & $0.3 \%$ \\
$r_{1}($ pole $)$ & $0.368 \pm 0.001$ & $0.367 \pm 0.001$ \\
$r_{1}$ (side) & $0.387 \pm 0.001$ & $0.386 \pm 0.001$ \\
$r_{1}($ back $)$ & $0.418 \pm 0.002$ & $0.416 \pm 0.001$ \\
$r_{2}($ pole $)$ & $0.348 \pm 0.003$ & $0.346 \pm 0.001$ \\
$r_{2}($ side $)$ & $0.366 \pm 0.004$ & $0.363 \pm 0.002$ \\
$r_{2}($ back $)$ & $0.398 \pm 0.006$ & $0.395 \pm 0.003$ \\
$\Sigma_{(\text {res })^{2}}$ & 0.0785 & 0.0137 \\
\hline $\mathcal{M}_{1} / \mathcal{M}_{\odot}$ & & 1.25 \\
$\mathcal{M}_{2} / \mathcal{M}_{\odot}$ & & $1.12^{*}$ \\
$R_{1} / R_{\odot}$ & & 2.32 \\
$R_{2} / R_{\odot}$ & & 2.20 \\
$\log \left(L_{1} / L_{\odot}\right)$ & & 0.91 \\
$\log \left(L_{2} / L_{\odot}\right)$ & & 0.67 \\
\hline
\end{tabular}

*assumed.

allows the adjustment of spot parameters. The results of the spotted solution are also given in Table 4 and the theoretical light curves are shown as solid lines in Fig. 4. The $\mathrm{O}-\mathrm{C}$ differences between the observed and calculated points for the unspotted and spotted solution for the system are shown in Fig. 5.

The parameters of the cool spot on the primary component are: latitude $b=90^{\circ}$ (fixed), longitude $l=271.27^{\circ} \pm$ $0.91^{\circ}$, angular radius $R=22.33^{\circ} \pm 4.51^{\circ}$ and temperature factor T.F. $=0.84 \pm 0.09$. Those of the hot spot are: latitude $b=90^{\circ}$ (fixed), longitude $l=21.26^{\circ} \pm 1.95^{\circ}$, angular radius $R=9.32^{\circ} \pm 2.69^{\circ}$ and temperature factor T.F. $=1.19 \pm 0.09$. A three dimensional picture of the spotted model at phases 0.25 and 0.75 is shown in Fig. 6, while the cross-sectional surface outline of the system together with the respective critical Roche lobes are given in Fig. 7.

\section{Conclusions}

The adopted spot model for YY CMi fits extremely well the observed light curves. The absolute elements based on its spectral classification and the present photometric solution are also given in Table 4 . We can use these elements to estimate the evolutionary status by means of 


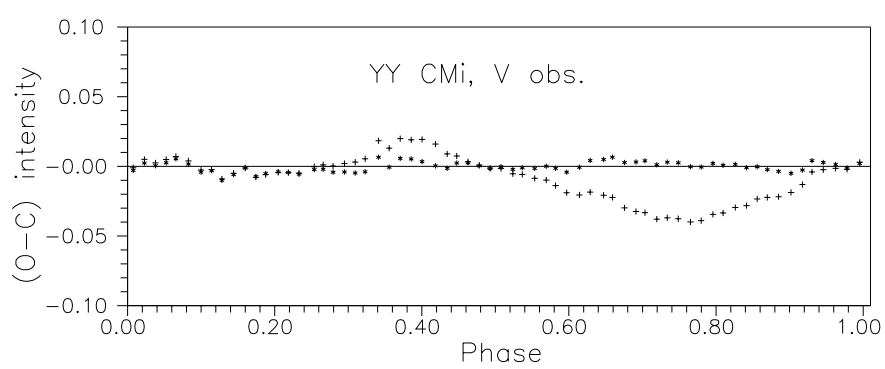

Fig. 5. The light curve $(\mathrm{O}-\mathrm{C})$ residuals for YY CMi in $V$ band. Crosses refer to unspotted solution; asterisks refer to spotted solution
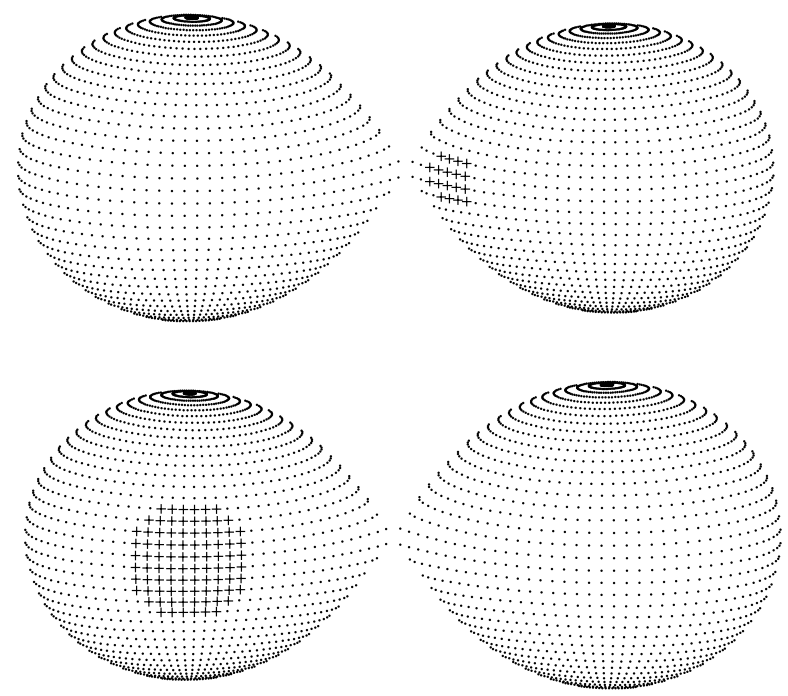

Fig. 6. A three-dimensional model of YY CMi for phases 0.25 (upper plot) and 0.75 (lower plot)

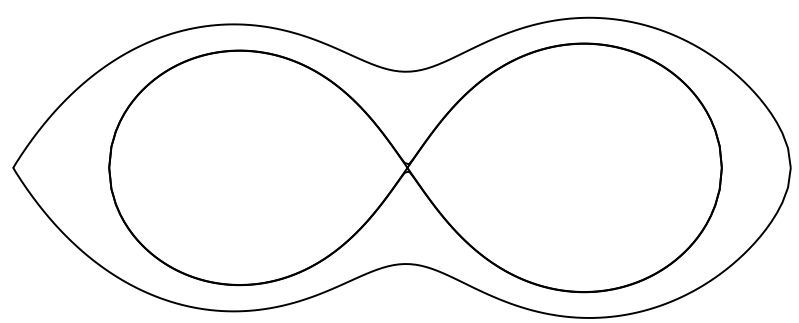

Fig. 7. Cross-sectional surface outline of YY CMi. It coincides with the inner Roche critical surface

the mass-radius (MR), mass-luminosity (ML) and HR diagrams of Hilditch et al. (1988). In these diagrams, both components of YY CMi lie beyond the TAMS, in a region occupied mostly by the primaries of A-type $\mathrm{W}$ UMa systems.
On the other hand, the degree of contact is almost zero (indicating marginal contact) and the thermal contact is poor $\left(T_{1}-T_{2}\right) \approx 650 \mathrm{~K}$. These results together with the high photometric mass-ratio $q \approx 0.88$ indicate that $\mathrm{YY}$ CMi is very probably a system at the beginning or the end of the contact phase (Lucy \& Wilson 1979). However, more definite conclusions about the evolutionary status of YY CMi can only be drawn by means of new photometric and spectroscopic observations of the system.

Acknowledgements. We thank the referee Dr. R.E. Wilson for his valuable comments on an earlier version of the manuscript. Figures 6 and 7 were produced by Binary Maker 2.0.

\section{References}

Abhyankar K.D., 1962, Z. Astrophysik 54, 25

Al-Naimiy H.M., 1978, ApSS 56, 219

Binnendijk L., 1960, AJ 65, 358

Bradstreet D.H., 1993, Binary Maker 2.0 User Manual

Giuricin G., Mardirossian F., 1981, A\&A 94, 391

Hilditch R.W., 1981, MNRAS 196, 305

Hilditch R.W., King D.J., McFarlane T.M., 1988, MNRAS 231, 341

Hill G., Hilditch R.W., Younger F., Fisher W.A., 1975, Mem. Roy. Astron. Soc. 79, 131

Kaho S., 1950, Tokyo Astr. Bull., 2nd Series, 30

Kholopov P.N., Samus' N.N., Frolov M.S., et al., 1985, General Catalogue of Variable Stars, IV Ed., v.I., "Nauka", 1985

Koch R.H., Plavec M., Wood F.B., 1970, A Graded Catalogue of Photometric Studies of Close Binaries, Univ. of Pennsylvania, Philadelphia

Kordylewski K., Szafraniec R., 1957, Acta Astron. 7, 177

Kukarkin B.V., Parenago P.P., Efremov Y.I., Kholopov P.N., 1969, Variable Star Catalogue, III Ed., Moscow

Lause F., 1938, Astr. Nachr. 266, 237

Linnell A.P., 1982, ApJS 50, 85

Maceroni C., Van Hamme W., van't Veer F., 1990, A\&A 234, 177

Mantegazza L., Poretti E., 1994, A\&A 281, 66

Milone E.F., Wilson R.E., Hrivnak B.J., 1987, ApJ 319, 325

Morgenroth O., 1934, Astr. Nachr. 252, 389

Mullan D.G., 1975, ApJ 245, 650

Soloviev A.V., 1940, Tadjek Circ. No. 43

Van Hamme W., 1993, AJ 106, 2096

Van Hamme W., Wilson R.E., 1985, A\&A 152, 25

van't Veer F., Maceroni C., 1988, A\&A 199, 183

van't Veer F., Maceroni C., 1989, A\&A 220, 128

Wilson R.E., 1990, ApJ 356, 613

Wood D.B., 1972, A Computer Program for Modeling NonSpherical Eclipsing Binary Systems, Greenbelt, U.S.A., Goddard Space Flight Center 